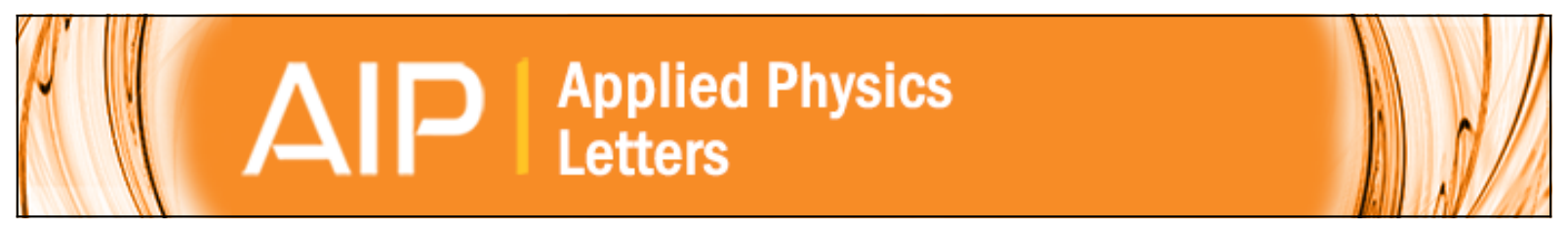

\title{
A small, linear, piezoelectric ultrasonic cryomotor
}

Shuxiang Dong, Li Yan, Naigang Wang, Dwight Viehland, Xiaoning Jiang, Paul Rehrig, and Wes Hackenberger

Citation: Applied Physics Letters 86, 053501 (2005); doi: 10.1063/1.1855424

View online: http://dx.doi.org/10.1063/1.1855424

View Table of Contents: http://scitation.aip.org/content/aip/journal/apl/86/5?ver=pdfcov

Published by the AIP Publishing

\section{Over 700 papers \& presentations on multiphysics simulation visw now}

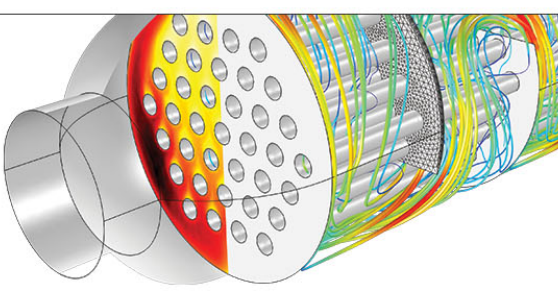




\title{
A small, linear, piezoelectric ultrasonic cryomotor
}

\author{
Shuxiang Dong, ${ }^{\text {a) }}$ Li Yan, Naigang Wang, and Dwight Viehland \\ Materials Science and Engineering, Virginia Tech, Blacksburg, Virginia 24061
}

Xiaoning Jiang, Paul Rehrig, and Wes Hackenberger

TRS Technologies, State College, Pennsylvania 16801

(Received 20 October 2004; accepted 3 December 2004; published online 24 January 2005)

\begin{abstract}
A small, linear-type, piezoelectric ultrasonic cryomotor has been developed for precision positioning at extremely low temperatures $\left(\geqslant-200^{\circ} \mathrm{C}\right)$. This cryomotor consists of a pair of $\mathrm{Pb}\left(\mathrm{Mg}_{1 / 3} \mathrm{Nb}_{2 / 3}\right) \mathrm{O}_{3}-\mathrm{PbTiO}_{3}$ single crystal stacks, which are piezoelectrically excited into the rotating third-bending mode of the cryomotor stator's center, which in turn drives a contacted slider into linear motion via frictional forces. The performance characteristics achieved by the cryomotor are: (i) a maximum linear speed of $>50 \mathrm{~mm} / \mathrm{s}$; (ii) a stroke of $>10 \mathrm{~mm}$; (iii) a driving force of $>0.2 \mathrm{~N}$; (iv) a response time of $\sim 29 \mathrm{~ms}$; and (v) a step resolution of $\sim 20 \mathrm{~nm}$. () 2005 American Institute of Physics. [DOI: 10.1063/1.1855424]
\end{abstract}

Piezoelectric motors operate by use of an ultrasonicwave/friction drive mechanism. They have high displacement resolution, and frictional locking in the power-off state; and they have the advantage of being driven by an electric field, without parasitic magnetic fields as for conventional electromagnetic motors. These merits make them good candidates for use in high-precision micromechanical systems. ${ }^{1-3}$ Various types of miniature ultrasonic motors have previously been reported ${ }^{4-13}$ that use a first-bending (B1) vibration mode based on $\mathrm{Pb}(\mathrm{Zr}, \mathrm{Ti}) \mathrm{O}_{3}$ (PZT) ceramic elements. However, the operational temperature range of PZT-based piezoelectric ultrasonic motors is limited in the range of $-40{ }^{\circ} \mathrm{C}$ (performance reduction and increased hysteretic losses) to $\sim 100{ }^{\circ} \mathrm{C}$ (thermal instability and depoling). For example, piezoelectric ceramics retain only $25 \%$ capability at a very low-temperature of $30 \mathrm{~K}$ and thus are not practical for low-temperature applications. This is unfortunate, as there are many applications requiring piezo-motors at temperatures outside of this operational window. One such example is cryogenic or space actuation applications. Recently, it was discovered that piezoelectric $\mathrm{Pb}\left(\mathrm{Mg}_{1 / 3} \mathrm{Nb}_{2 / 3}\right) \mathrm{O}_{3}-x \% \mathrm{PbTiO}_{3}(\mathrm{PMN}-x \% \mathrm{PT})$ and $\mathrm{Pb}\left(\mathrm{Zn}_{1 / 3} \mathrm{Nb}_{2 / 3}\right) \mathrm{O}_{3}-x \% \mathrm{PbTiO}_{3}(\mathrm{PZN}-x \% \mathrm{PT})$ single crystals have excellent actuation characteristics at cryogenic temperatures. ${ }^{14,15}$ Stacks of these piezoelectric crystals operated at $30 \mathrm{~K}$ have shown piezoelectric properties superior to that of piezoelectric ceramics at $300 \mathrm{~K}$.

In this study, we have developed a notched-type composite piezoelectric stator. Prototypes were made from two single crystal PMN-PT ring stacks and a stainless steel bar. Each PMN-PT stack consisted of four PMN-PT rings; each single crystal ring had coated on it a two segment electrode and under which the PMN-PT crystal segments were poled in reverse directions with respect to each other. Upon application of drive voltage to one of these ring stacks, a bending vibration is generated in one-direction-one segment expands, whereas the other contracts. Two such ring stacks were assembled orthogonally in the stator. This results in two orthogonal bending vibration modes, as illustrated in Fig. 1. Upon application of two sine drives to the two PMN-PT ring

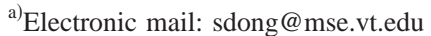

stacks (phase shifted by $90^{\circ}$ ), a rotating/bending vibration (i.e., a wobbling motion) can be excited either at the ends or center of the stator. The role of the two notches in the stator is to decrease the effective bending stiffness at the stator's center. As a result, the stator's center has enhanced bending vibrations relative to its ends, in particular when operated in its third-bending (B3) vibration/bending mode. Analysis using ATILA (Analysis of Transducers by Integration of Laplace equations ${ }^{16}$ ) finite element method (FEM) software illustrates the displacement amplification offered by this drive mechanism concept. In Fig. 2, excitation of the two bending vibrations of the two ring stacks (shown as down direction) results in a much larger bending vibration at the stator's center (shown as up direction). Clearly, enhancement of the vibration amplitude is achieved at the center-point of the notched composite stator when it is operated in B3 mode. This notched piezoelectric stator operated in its B3 bending mode is notably different from conventional rod-type ones operated in their B1 mode, ${ }^{4-13}$ and could potentially be used for a new slider drive mechanism.

Figure 3 illustrates the construction and working principle of our linear center-wobbling piezoelectric stator. The stator's center was elastically pressed together in order to ensure frictional contact with the slider. The slider was then excited into linear motion, by contact to the center-wobbling motion of the stator. Our prior experimental results reveal that the driving force of this center-wobbling mode is much stronger than that offered by an end-wobbling one. ${ }^{12}$

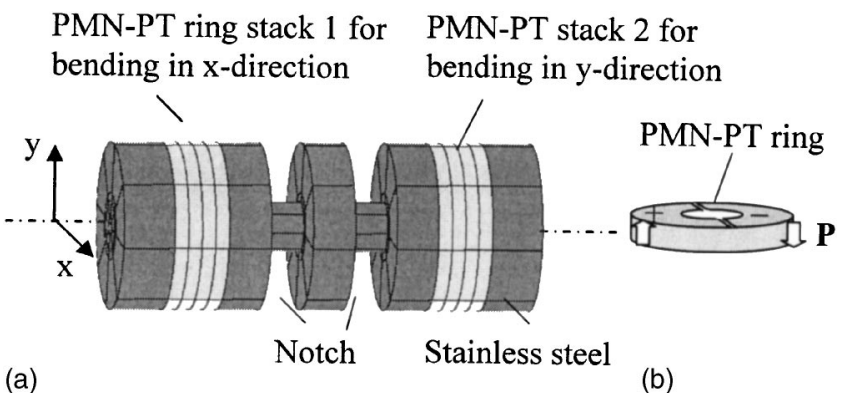

FIG. 1. Construction of PMN-PT piezoelectric composite stator: (a) piezocomposite stator with two notches and (b) a single PMN-PT ring. The symbol $\mathbf{P}$ indicates the polarization direction. 


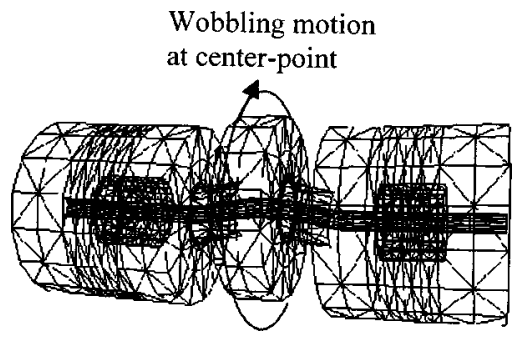

FIG. 2. B3 wobbling mode of the stator, calculated using Atila FEM software code.

The stator was a notched cylinder with a diameter of 10 $\mathrm{mm}$ and a length of $25 \mathrm{~mm}$. The two single crystal piezoelectric ring stacks (each consisting of four single crystals disks) were $10 \mathrm{~mm}$ in outer-diameter, $3.5 \mathrm{~mm}$ in innerdiameter, and $4 \mathrm{~mm}$ in thickness. Rings were placed at both ends of the stator. Gold electrodes were sputtered onto the PMN-PT single crystal rings, which were patterned into two segments. A room temperature two-step poling procedure was then followed. During the first step, one segment was poled under a quasistatic field of $E=10 \mathrm{kV} / \mathrm{cm}$ at frequency of $f<0.01 \mathrm{~Hz}$, while the other segment was shorted to ground and in the second step, the second segment was poled in the reverse direction by similar steps. A cryogenic epoxy was used to bond the single crystal disks and metal shims (including end-caps) together. The resonant mode of the assembled stator was measured using an impedance analyzer. A distinct B3 wobbling mode with a resonant frequency of $\sim 41.5 \mathrm{kHz}$ was found at room temperature. On cooling to 77 $\mathrm{K}$, this mode's resonance frequency was increased to $\sim 45 \mathrm{kHz}$.

First, the main performance characteristics of an assembled, small, linear, ultrasonic motor were experimentally determined. Using a pair of drive voltages of $60-70 \mathrm{~V}_{\mathrm{pp}}$ (phase shifted by $90^{\circ}$ ) that were applied to the stator, the traveling speed of the slider was found to be $50-100 \mathrm{~mm} / \mathrm{s}$. By applying weight to the stator, the maximum load (driving force) of the motor was determined to be $\sim 0.2 \mathrm{~N}$.

Next, the step displacement of our ultrasonic motor was measured by applying signals of various pulse widths and cycle periods in both forward and backward directions. A function generator and wave writer (AFG320) was used to generate a control pulse. This pulse then triggered a twophase output driving circuit, which generated and controlled the motor's stepping displacement range. An optical-fiber sensor (MTI2000 Fotonic Sensor) and digital oscilloscope (54624A, Agilent) were used to record the stepping displacements and response time. Figures 4(a) and 4(b) show the linear motion using step displacements of 79 and $21 \mathrm{~nm}$, respectively. Scatter in the data is due to background vibration noise, which could be eliminated by testing on a vibration isolation table. The response time of the linear motor

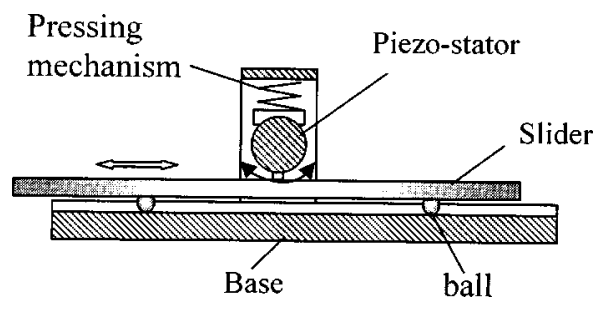

FIG. 3. Piezoelectric linear motor, operated in its B3 wobbling mode.
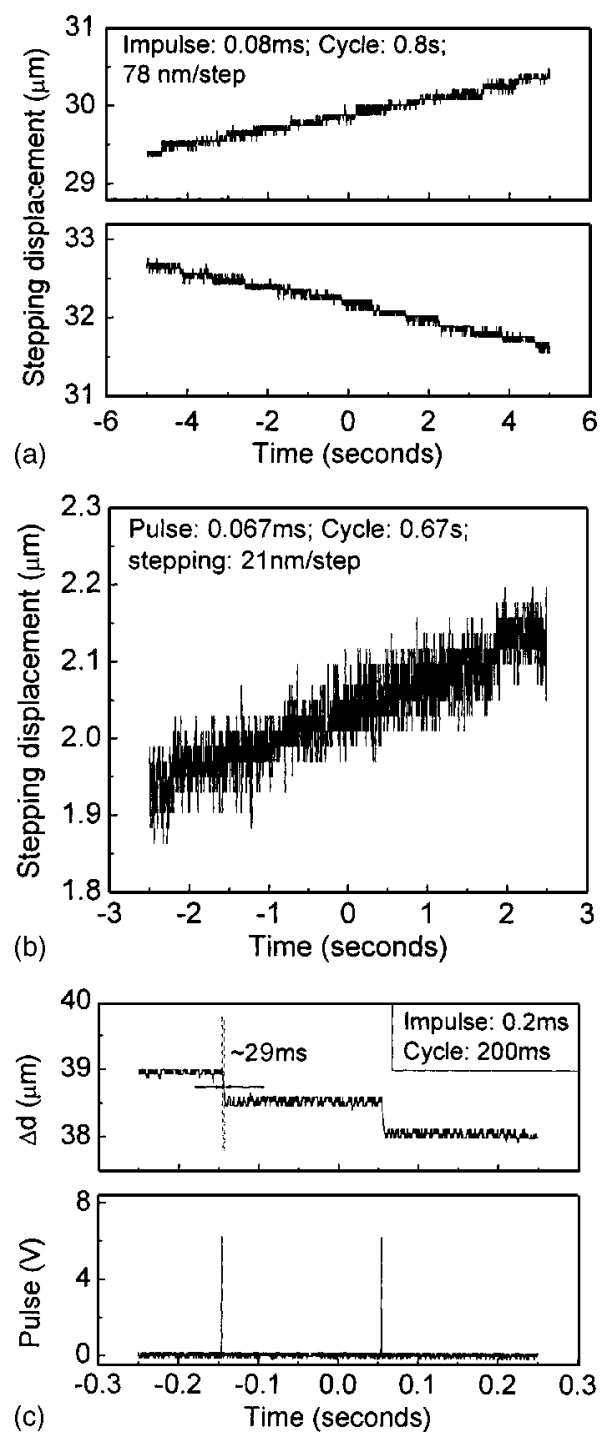

FIG. 4. Characteristics of the linear motor: (a) stepping motion at $78 \mathrm{~nm} / \mathrm{step}$ in forward and backward directions; (b) stepping motion at $21 \mathrm{~nm} / \mathrm{step}$ in forward and backward directions; and (c) response time of the linear motor to a pulse control signal.

was determined by applying a square-wave voltage pulse of sharp impulse, and by subsequently recording the timedependent displacement response. The response time of our linear ultrasonic motor was $\sim 29 \mathrm{~ms}$, as shown in Fig. 4(c). This is as fast as other types of linear ultrasonic motors. ${ }^{15}$ Furthermore, our linear ultrasonic motor demonstrated not only a quick response time from the start/stop conditions, but also to reversals in the direction of motion.

Finally, we tested the cryogenic capabilities of our linear ultrasonic motor. The motor was placed in an environmental test chamber and cooled by liquid nitrogen to $\sim 77 \mathrm{~K}$. The experimental results confirmed that the motor worked properly at these cryogenic temperatures, suffering only a slight decrease in its speed.

In summary, a center-wobbling, single-crystal, lineartype ultrasonic cryomotor has been developed with characteristics of (i) high precision, offering a step resolution of $\sim 20 \mathrm{~nm}$; (ii) a large stroke of $>10 \mathrm{~mm}$, which in principle is not limited from being higher; and (iii) a rapid response time of $\sim 29 \mathrm{~ms}$. In addition, successful performance of a motor prototype has been demonstrated over the temperature range 
of $77<T<300 \mathrm{~K}$-offering promise for cryogenic actuation applications.

This work was sponsored by NASA under Grant No. NC04CA87C. The authors thank Dr. Benjamin C. Platt and Dr. Steven Gunter from NASA-JPL for project technical monitoring and helpful discussions. Hua Lei of TRS Technologies is also gratefully acknowledged for help in stack assembly.

${ }^{1}$ S. Ueha and Y. Tomikawa, Ultrasonic Motors: Theory and Applications (Clarendon, Oxford, 1993), pp.4-6.

${ }^{2}$ T. Sashida and T. Kenjo, An Introduction to Ultrasonic Motors (Clarendon, Oxford, 1993), pp.6-8.

${ }^{3}$ K. Uchino, Piezoelectric Actuators and Ultrasonic Motors (Kluwer Academic, Boston, 1997).

${ }^{4}$ W. Williams and W. J. Brown, U.S. Patent 2439499, April 13, 1948.

${ }^{5}$ J. Wallaschek, J. Intell. Mater. Syst. Struct. 6, 71 (1995).

${ }^{6}$ I. Okamura (Private communication).
${ }^{7}$ M. Kurosawa, K. Nakamura, T. Okamoto, and S. Ueha, IEEE Trans. Ultrason. Ferroelectr. Freq. Control 36, 517 (1989).

${ }^{8}$ S. Dong, S. Wang, W. Shen, and L. Li, IEEE/ASME Trans. Mechatron. 5, 325 (2000).

${ }^{9}$ T. Morita, M. Kurosawa, and T. Higuchi, IEEE Trans. Ultrason. Ferroelectr. Freq. Control 40, 687 (1993).

${ }^{10}$ T. Morita, M. Kurosawa, and T. Higuchi, Jpn. J. Appl. Phys., Part 1 38, 3347 (1999).

${ }^{11}$ S. Dong, S. P. Lim, K. H. Lee, J. Zhang, L. C. Lim, and K. Uchino, IEEE Trans. Ultrason. Ferroelectr. Freq. Control 50, 361 (2003).

${ }^{12}$ S. Dong, S. Cagatay, K. Uchino, and D. Viehland, J. Intell. Mater. Syst. Struct. 13, 749 (2002).

${ }^{13}$ H. Koc, S. Cagatay, and K. Uchino, IEEE Trans. Ultrason. Ferroelectr. Freq. Control 49, 495 (2002).

${ }^{14}$ S. E. Park, V. Vedula, M. J. Pan, W. S. Hackenberger, P. Pertsch, and T. R. Shrout, SPIE Conference on Smart Materials and Technologies, San Diego, CA, March, 1998, Vol. 3324.

${ }^{15}$ D. S. Paik, S. E. Park, W. S. Hackenberger, and T. R. Shrout, J. Mater. Sci. 34, 469 (1999).

${ }^{16}$ Atila operational manual 1998, Magsoft Corporation. 